\title{
Antenatal diagnosis of microvillus inclusion disease
}

\begin{abstract}
Microvillus inclusion disease is a rare autosomal recessive disorder due to defective apical surface of the enterocytes presenting with severe watery diarrhea starting at birth. We describe a female infant who had antenatal diagnosis of microvillus inclusion disease. At 36 th gestational week of a 32-year-old woman ultrasound examination revealed dilatation of fetal sigmoid colon. The amniotic fluid level was normal. An amniocentesis was performed to rule out congenital sodium and chloride diarrhea in the prenatal period. The patient didn't prefer to undergo genetic tests. In conclusion, prenatal ultrasonographic identification of dilated bowel loops without polyhydramnios suggests differential diagnosis of microvillus inclusion disease in addition to congenital chloride diarrhea, jejunoileal atresia, volvulus, meconium ileus, Hirschsprung disease, enteric duplications, anorectal atresia.
\end{abstract}

Keywords: congenital diarrhea, microvillus inclusion disease, prenatal diagnosis
Volume 12 Issue 4 - 202I

\author{
Gular Israfilova, Banu Arslanca, Yavuz Emre \\ Sukur,Acar Koç \\ Department of Obstetrics and Gynecology,Ankara University \\ School of Medicine, Turkey
}

\begin{abstract}
Correspondence: Gular Israfilova, MD,Ankara University School of Medicine, Department of Obstetrics and Gynecology, Dikimevi,Ankara, Turkey, Tel 00905375752340 ,
\end{abstract}

Emaildrisrafilova@gmail.com

Received: July 29, 2021 | Published: August 12, 2021

\section{Introduction}

Microvillus inclusion disease (MVID) is a congenital bowel disease characterized by severe diarrhea, malabsorption and growth retardation in infancy. ${ }^{1}$ Severe watery diarrhea begins in the first days of life, with the loss of $30 \%$ of body mass in the first 24 hours. Metabolic acidosis and hypotonic dehydration develop in children with MVID. ${ }^{2}$ Impaired kidney function, accompanied by nephrocalcinosis is a common complication. ${ }^{3}$ Children with MVID are dependent on parenteral nutrition throughout their lives, and most of these children are at risk of developing cholestasis and liver failure due to total parenteral nutrition (TPN).

The diagnosis of MVID is based on an intestinal biopsy. Light microscopy is characterized by accumulation of Periodic acid-Schiff (PAS) positive granules in immature enterocytes in upper crypts. ${ }^{4}$ There is no curative treatment for MVID. Trials of anti-inflammatory drugs, including steroids and anti-secretory agents, have not succeeded in significantly altering stool volumes over a long period. ${ }^{5}$ We describe a case of MVID in which antenatal ultrasound revealed structural deformities. A written informed consent was obtained from the patient before preparing for publication.

\section{Case presentation}

A 32-year-old G1 P0 woman was diagnosed to have dilatation of fetal sigmoid colon on prenatal ultrasound at $36^{\text {th }}$ gestational weeks (Figure 1). The amniotic fluid index was $15 \mathrm{~cm}$. The parents were not consanguineous. First trimester screening and level 2 ultrasound results were normal. Anal atresia was ruled out based on the absence of $\mathrm{U}$ or $\mathrm{V}$-shaped intestine in the pelvis, lack of enterolithiasis, condensation or meconium accumulation on prenatal ultrasounds at 24, 28, 32 and 34 weeks. Amniocentesis was performed to exclude congenital chloride diarrhea. The electrolytes were as follows: Na: $122, \mathrm{~K}: 3.8, \mathrm{Cl}: 101$, osmolarity: $240 \mathrm{mOsm} / \mathrm{kg}$. The lack of fluiddebris levels, non-dilatation of the small intestine, and monitoring of bowel peristalsis in the coronal ultrasonography helped to rule out volvulus. The preliminary diagnoses were Hirschsprung's disease and microvillus inclusion disease.

The patient gave birth to a $3420 \mathrm{~g}$ female vaginally at $40^{\text {th }}$ gestational week. The APGAR scores at $1^{\text {st }}$ and $5^{\text {th }}$ minutes were 7 and 9 , respectively. On postpartum $3^{\text {rd }}$ day, the neonate suffered from watery diarrhea and abdominal distension. Abdominal X-ray showed dilated intestinal loops and pneumoperitoneum (Figure 2). On postpartum $5^{\text {th }}$ day, the infant was referred to the gastroenterology department due to $19 \%$ weight loss. An endoscopic biopsy revealed intra-cytoplasmic inclusions in the apical surface of the intestinal epithelial cells and villus loss, with a CD10 and PAS epithelial cytoplasmic staining pattern supporting the diagnosis of MVID.

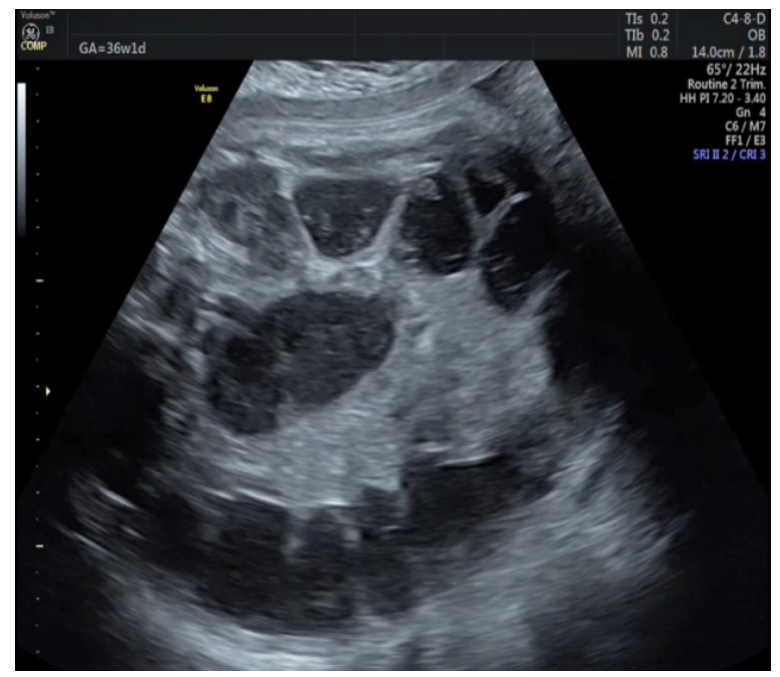

Figure I Prenatal ultrasound at $36^{\text {th }}$ gestational week showing fluid-filled large bowel.

The patient was placed on a central venous catheter due to the need for long-term total parenteral nutrition (TPN). Coagulase-negative staphylococci were produced in blood culture. Computed tomography revealed ascites, mucosal enhancement of the stomach and bowel loops, increased heterogeneous contrast in liver parenchyma, patched ground-glass areas in the lungs, inter-lobular septum thickening and bilateral pleural effusions potentially associated with hypoproteinemia and volume overload.

The infant died due to septic shock secondary to a catheter-related infection. The post-mortem genetic examination revealed compound 
heterozygous mutation of MYO5B gene. Additionally, heterozygous presence of a variant of unknown significance (VOUS) in MYO58 was also identified. It was determined c.1355_1363 dup heterozygosis mutation in MYO5B gene in paternal blood sample and $1463>$ Cand p.lle $488 \mathrm{Thr}$ heterozygosis mutation in gene MYO5 in maternal blood sample.

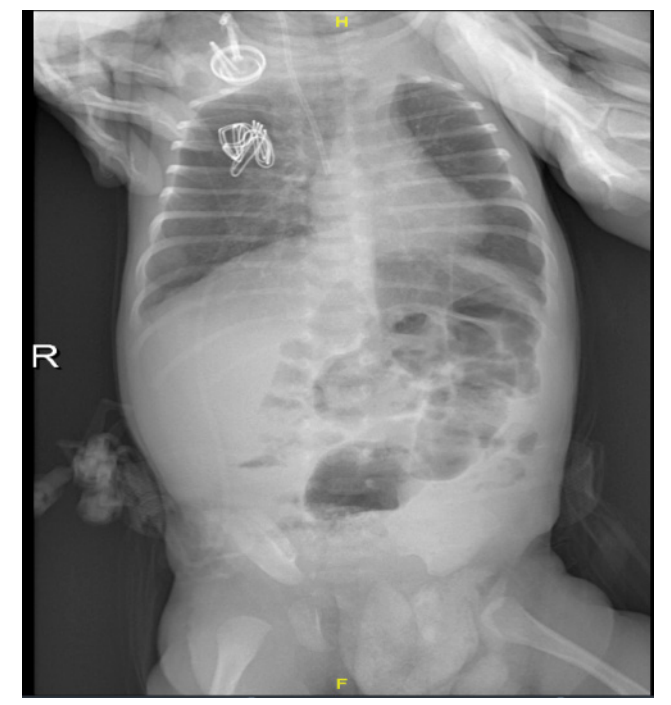

Figure 2 Dilate intestinal loops and pneumoperitoneum in newborn.

\section{Discussion}

Microvillous inclusion disease is an autosomal recessive disease and usually caused by mutations in $M Y O 5 B$. It presents with chronic, watery, life-threatening diarrhea which results in severe dehydration and metabolic acidosis. ${ }^{6}$ The prognosis of disease is very poor and usually fatal as a result of cholestatic liver disease or from sepsis. Today, a prenatal diagnosis of MVID is also available based on molecular genetics.

Differential diagnosis of MVID should be done for jejunoileal atresia, volvulus, meconium ileus, Hirschsprung's disease, enteric duplications, anorectal atresia, congenital salt loss diarrhea, Bartter syndrome and enteroendocrine cell dysgenesis. Anal atresia was ruled out based on absence of $\mathrm{U}$ or V-shaped intestine in the pelvis, lack of enterolithiasis, condensation or meconium accumulation and visualization of anus on prenatal ultrasound. The lack of fluiddebris levels, non-dilatation of the small intestine, and monitoring of bowel peristalsis in the coronal ultrasonography helped to rule out volvulus. Enteroendocrine cell dysgenesis can be differentiated from microvillous inclusion disease by the lack of enteroendocrine cells and the presence of normal microvilli. An antenatal differential diagnosis algorithm based on prenatal ultrasound findings for MVID has not been published yet. Figure 3 shows an algorithm created by the authors for the differential diagnosis of dilated intestinal loops at prenatal ultrasound.

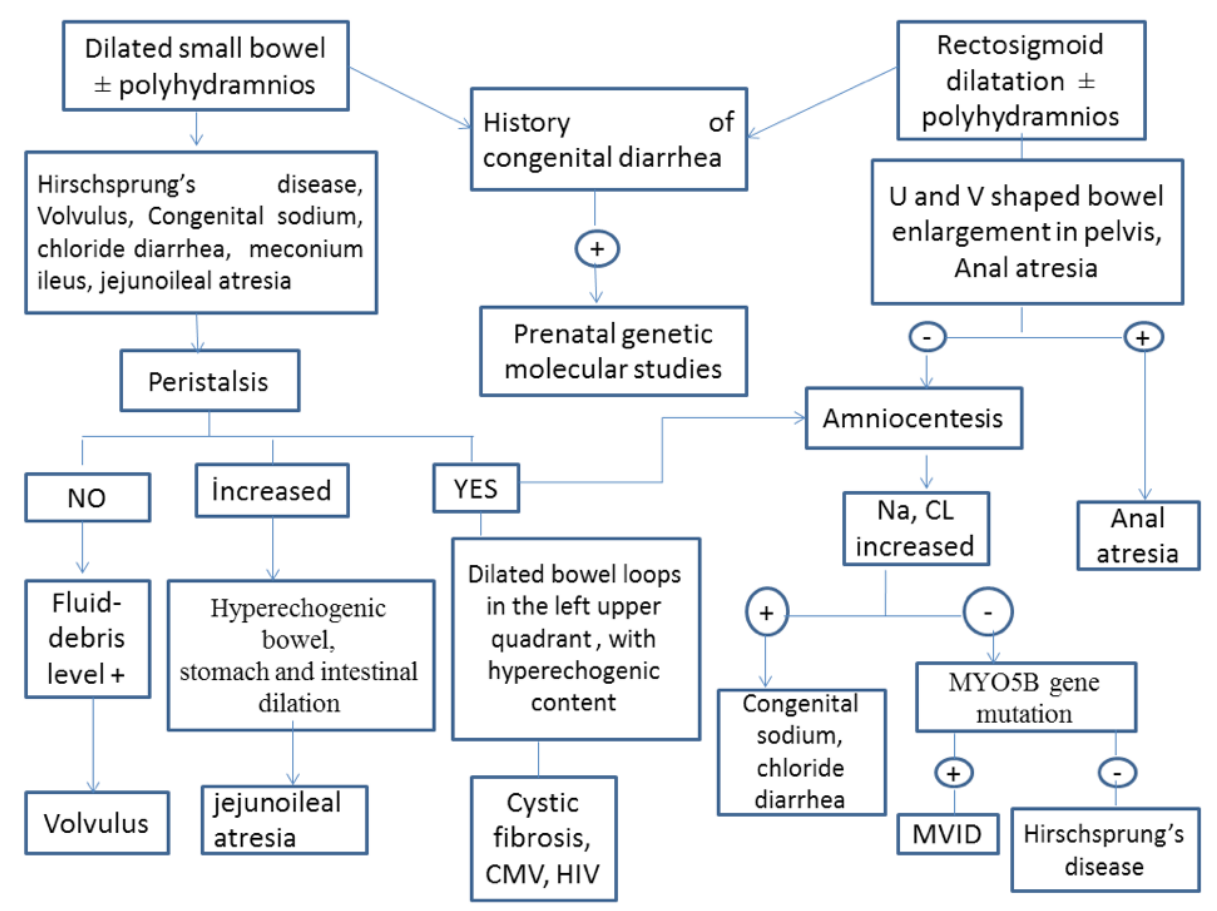

Figure 3 Differential diagnosis of dilated intestinal loops at prenatal ultrasound.

Several previous reports suggested excluding jejunoileal atresia, volvulus, meconium ileus, Hirschsprung disease, enteric duplications, anorectal atresia, congenital salt loss diarrhea, Bartter syndrome, and MVID from differential diagnoses when dilated intestines are detected on prenatal ultrasound..$^{7-9}$ Fetuses with MVID may present with multiple dilated bowel loops and polyhydramnios mimicking intestinal obstruction in the early third trimester.?
Congenital chloride and sodium diarrheas may also present with polyhydramnios multiple dilated bowel loops, fluid filled intestinal obstruction on prenatal ultrasound and elevated amniotic fluid electrolyte concentrations. ${ }^{8,10}$

Kennea et al. ${ }^{9}$ reported that polyhydramnios accompanied by dilated large and small bowel loops which resembled an intestinal 
obstruction on antenatal ultrasound could lead to a postnatal diagnosis of MVID. ${ }^{9}$ The authors stated that bowel obstruction and congenital enteropathy should be considered if a prenatal ultrasound showed dilated bowel loops and polyhydramnios. In the present case, amniocentesis was helpful to rule out the diagnosis of congenital sodium and chloride diarrheas in the prenatal period.

Currently, there is no curative treatment for MVID. Although longterm TPN or bowel and liver transplantation are treatment options, MVID-related complications and reduced longevity are common. Novel genome editing technologies, such as CRISPR/Cas9, hold promise for MVID treatment. However, further studies are needed to shed light on. ${ }^{11}$

\section{Conclusion}

When dilated bowel loops are detected regardless of the presence of polyhydramnios in prenatal ultrasound examination, MVID should be considered as a differential diagnosis. Prenatal genetic molecular studies should be performed to confirm the diagnosis of MVID.

\section{Acknowledgments}

None.

\section{Funding}

None.

\section{Conflicts of interest}

No potential conflicts of interest are reported by the authors.

\section{References}

1. Davidson GP, Cutz E, Hamilton JR, et al. Familial enteropathy: a síndrome of protracted diarrhea from birth, failure to thrive and hipoplastic villous atrophy. Gastroenterology. 1978;75:783-790.
2. Golachowska M, van Dael ML, Keuning H, et al. MYO5B mutations in patients with microvillus inclusions disease presenting with transient renal Fanconi syndrome. Journal of pediatric gastroenterology and nutrition. 2012;54:491-498.

3. Siahanidou T, Koutsounaki E, Skiathitou A, et al. Extraintestinal manifestations in an infant with microvillus inclusion disease: complications or features of the disease? European journal of pediatrics.2013;172:1271-1275.

4. Phillips AD, Szfranski M, Man L-Y, et al. Periodic acid Schiff staining abnormality in microvillous atrophy: photometric and ultrastructural studies. J Pediatr Gastroenterol Nutr. 2000;30:34-42.

5. Phillips AD, Schmitz J. Familial microvillous atrophy: a clinicopathological survey of 23 cases. J Pediatr Gastroenterol Nutr. 1992;14:380-396.

6. Berni Canani R, Terrin G, Cardillo G, et al. Congenital diarrheal disorders: improved understanding of gene defects is leading to advances in intestinal physiology and clinical management. J Pediatr Gastroenterol Nutr 2010;50:360-366.

7. Chen $\mathrm{C}$, Chiang $\mathrm{M}$, Wang $\mathrm{T}$, et al. Microvillus inclusion disease: prenatal ultrasound findings, molecular diagnosis and genetic counseling of congenital diarrhea. Tai wan J Obstet Gynecol. 2010;49(4):487-494.

8. Langer JC, Winthrop AL, Burrows RF, et al. False diagnosis of intestinal obstruction in a fetus with congenital chloride diarrhoea. J Ped Surg. 1991;26:1282-1284.

9. Kennea N, Norbury R, Anderson G, et al. Congenital microvillous inclusion disease presenting as antenatal bowel obstruction. Ultrasound Obstet Gynecol 2001;17:172-174.

10. Colombani M, Ferry M, Toga C, et al. Magnetic resonance imaging in the prenatal diagnosis of congenital diarrhea. Ultrasound Obstet Gynecol. 2010;35:560-565.

11. Vogel GF, Michael WH, Lukas KP, et al. Towards understanding microvillus inclusion disease. Molecular and cellular pediatrics. 2016;3:3. 